\title{
Research on the Delivery System of infectious diseases vaccine
}

\author{
Lei Li
}

School of Electric Power Engineering, North China Electric Power University, Baoding 071000, China;

1010898691@qq.com

Keywords: Fuzzy clustering, Knapsack problem.

\begin{abstract}
In this paper, distribution is also what we are concerned with. We consider the situations where the drug supply is unlimited and where the drug supply is limited. With the help of the fuzzy clustering analysis and the knapsack problem model, we get the optimal solution to the limited drugs supply. As for the unlimited supply, by selecting transfer points, we build a feasible delivery system.
\end{abstract}

\section{Introduction}

We take the delivery system, the transportation and the production rate of drugs into account. Based on the consideration of different production rates, we establish two delivery systems to distribute drugs in a most efficient way.

\section{Assumptions}

- The natural birth rate is approximately equal to mortality and the net migration rate is approximately equal to zero.

- The current virus hasn't varied during this short period of time.

- We assume the recovered person wouldn't be infected again. As the previous cases shows, there isn't anyone, who has recovered from Ebola, being infected again.

- We don't consider the effects between countries.

\section{Parameters}

Define sample set $U=\left\{x_{1}, x_{2}, \ldots, x_{n}\right\}$ as objects to be classified, and each object is indicated by 3 indexes: $x_{i}=\left\{x_{i 1}, x_{i 2}, x_{i 3}\right\}$

Table 1 Symbols and Definitions

\begin{tabular}{cc}
\hline Symbol & Definition \\
\hline$x_{i 1}\left(1 \leq x_{i 1} \leq 5\right)$ & The grade of each district on "confirmed cases". \\
$x_{i 2}\left(1 \leq x_{i 2} \leq 5\right)$ & The grade of each district on "cases in past 21 days". \\
$x_{i 3}\left(1 \leq x_{i 3} \leq 6\right)$ & The grade of each district on "days since last case”. \\
\hline
\end{tabular}

\section{Delivery System}

Assuming that the infected areas are in Africa.[1] As the medical manufactories producing drugs are outside the African continent, drugs can be transported to Africa in two ways: by sea or by air. [2]

(1) By sea: huge capacity for carrying with quite slow speed.

(2) By air: less capacity for carrying with much faster speed.

Considering drugs should be distributed to the epidemic areas as soon as possible, all the drugs are transported to the distribution centers of drugs by air, which are the capitals of the countries.

Since the real production rate of the drug is unclear, we need to consider how to distribute the drugs in the case that the supply is unlimited and the case that the supply is limited. 


\section{Unlimited Supply}

As the purpose of the drug distribution is to eliminate the virus, any demand for the drugs should be immediately met regardless of the cost when the supply is unlimited.

Although the supply of drugs is unlimited, because these areas are spread across all over the country, sending the drug transport vehicle to every point of demand directly is impossible. Therefore, we need to establish transfer stations of distribution in different regions so as to meet the drug distribution of some remote areas.

The establishment of the transfer stations of distribution should meet some conditions [3] as follows:

- The distance to distribution center, namely capital, is at least $100 \mathrm{~km}$, otherwise, the transfer station of distribution is meaningless.

- The traffic in the transfer station should be convenient to meet that vehicles from all directions can approach to the transfer station.

Generally speaking, the size of the city can reflect the level of a city's transportation convenience. Therefore, we use the size to characterize the level of local transportation convenience.

The rules of drug distribution are as follows:

1. First, transit drugs to the distribution center intensively through the air. And then, choose the nearest distribution center as the source of their drugs, through calculating the distance of each transfer station and distribution center by using GPS system.

2. Because they are tightly sealed in goods van when transported by trucks, drugs can't be distributed before arriving in transfer station.

3. Every point of demand chooses the nearest distribution center as the source of their drugs through calculating the distance to each near distribution center by using GPS system.[3]

\section{Limited Supply}

Subject to the low production rate, it is quite possible that the supply of the drugs is insufficient. We should consider two possible allocation plans:

$>$ Distribute the drugs according to their demand fairly; which means that the case fatality rate and the infection rate of each district will decrease by the same degree.

$>$ Give the priority to the districts where the epidemic situation is much more severe(more infected people), which means that the case fatality rate and the infection rate of the district given the priority will decrease a lot while other districts decrease a little or remain the same. Figure 1 shows the difference between equal allocation and priority allocation.

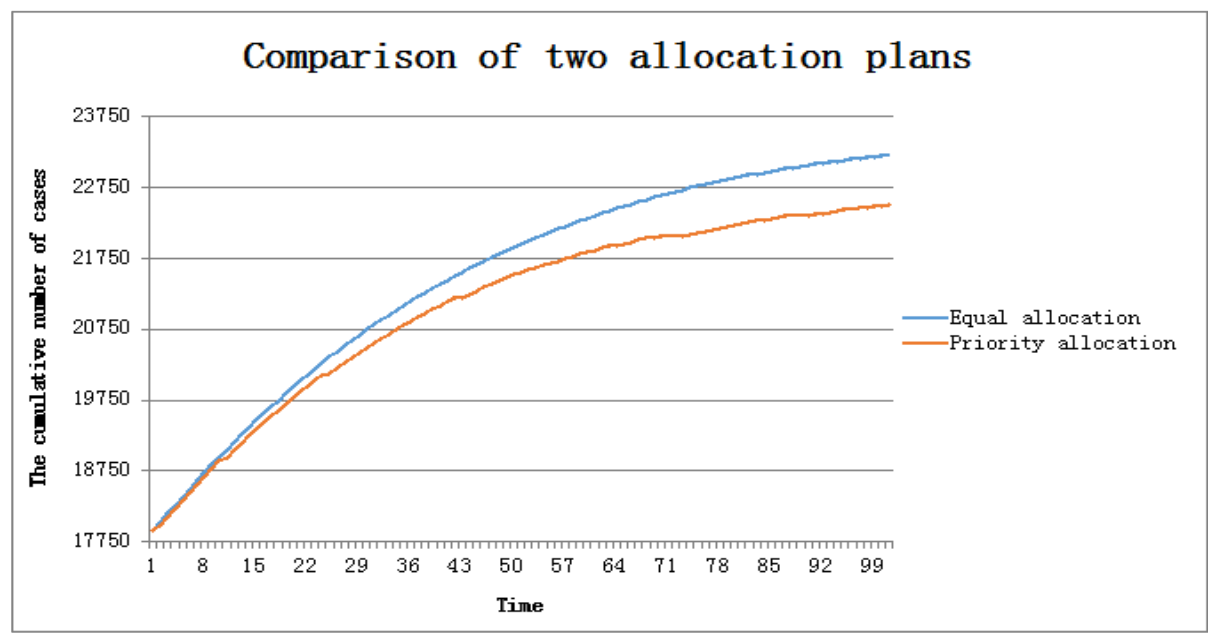

Fig. 1 Comparison of two allocation plans

\section{Distribution Model Establishment}

$A_{i}$ represents different demand districts respectively. By using fuzzy clustering analysis [3], we can divide the districts into different groups based on several classification indicators (confirmed cases, cases in past 21 days, and days since last case). Situations of each district in the same group are as similar as possible while situations between different groups are as dissimilar as possible. After the 
grouping, we can give different priorities to different groups on the basis of the current situation.

Sequence of the drug distribution is in accordance with the priority, in other words, districts with higher priority will get the drugs first and then districts with the next priority can get drugs. If drugs are not enough in some priority, we use the internal distribution model to decide how to distribute in that priority (also called the cut-off priority). However, districts with priority lower than the cut-off priority may not get any drug.

\section{Cluster analysis}

(1) Initial matrix:

By collecting the statistics of each district's cluster indexes, we can get the following table:

Table 7:

\begin{tabular}{|c|c|c|c|}
\hline District index & $I_{1}$ & $I_{2}$ & $I_{3}$ \\
\hline$D_{1}$ & $x_{11}$ & $x_{12}$ & $x_{13}$ \\
\hline$D_{2}$ & $x_{21}$ & $x_{22}$ & $x_{23}$ \\
\hline$D_{3}$ & $x_{31}$ & $x_{32}$ & $x_{33}$ \\
\hline$\cdots \cdots$ & $\cdots \cdots$ & $\cdots \cdots$ & $\cdots \cdots$ \\
\hline
\end{tabular}

Extract elements from the table, and we can get the initial matrix by putting them together.The initial matrix [3] is:

$$
\left[\begin{array}{ccc}
x_{11} & x_{12} & x_{13} \\
x_{21} & x_{22} & x_{23} \\
x_{31} & x_{32} & x_{33} \\
\vdots & \vdots & \vdots
\end{array}\right]
$$

(2) Data standardization:

First of all, standardize the cluster indexes. [4] Transformation formula is as follows:

$$
x_{i j}^{\prime}=\frac{1}{t_{j}}\left(\mathrm{x}_{i j}-\overline{x_{j}}\right)
$$

Where:

$$
\begin{aligned}
& \overline{x_{j}}=\frac{1}{n} \sum_{i=1}^{n} x_{i j} \\
& t_{j}=\sqrt{\frac{1}{n} \sum_{i=1}^{n}\left(x_{i j}-\overline{x_{j}}\right)^{2}}
\end{aligned}
$$

Next, use range transformation to insure that all the data are distributed within the interval $[0,1]$. Transformation formula[2] is as follows:

$$
x^{\prime \prime}{ }_{i j}=\frac{x_{i j}^{\prime}-\min _{i s i s n}\left\{x_{i j}^{\prime}\right\}}{\max _{i s i s n}\left\{x_{i j}^{\prime}\right\}-\min _{i s i s n}\left\{x_{i j}^{\prime}\right\}}
$$

(3) Establish the fuzzy similar matrix [4]:

With subtrahend's absolute value method, we can get the fuzzy similar matrix $S$.

$$
s_{i j}=\left\{\begin{array}{c}
1, i=j \\
1-c \sum_{k=1}^{m}\left|x_{i k}-x_{j k}\right|, \mathrm{i} \neq \mathrm{j}
\end{array} \text { and } s=\left[s_{i j}\right]_{m \times n}\right.
$$

Where $c$ should be assigned properly so that $s_{i j}$ can be distributed within the interval $[0,1]$.

If the fuzzy similar matrix $S$ is of transitivity ( $S^{\circ} S \subseteq S$ ), then we can customize a threshold value $\lambda$ that can divide the sample set $\mathrm{U}$ into different groups; however, if the fuzzy similar matrix $S$ is not of transitivity, then we need to apply the transfer closure method to get the fuzzy equivalent matrix $C$, and replace the fuzzy similar matrix $S$ with the fuzzy equivalent matrix $C$ before we customize a threshold value $\lambda$ to divide the sample set $U$ into different groups. Matrix $C$ is 


$$
C=A^{\circ} B
$$

Where

$$
\begin{aligned}
& A=\left[a_{i k}\right](i=1,2, \ldots, m ; j=1,2, \ldots, l) \\
& B=\left[a_{k j}\right](k=1,2, \ldots, l ; j=1,2, \ldots, n) \\
& C=\left[c_{i j}\right](i=1,2, \ldots, m ; j=1,2, \ldots, n)
\end{aligned}
$$

After the Cluster analysis, we can get the district priority ranking.

\section{References}

[1] Dao Tianxi, XuShoujun, Zhao Xiaoyu, Zhou Wei, Li Peng, Liu Shu and Wang Wei, Ebola fever drugs and analysis of vaccine research and development trend[J]. Military medicine, (08): 569-575, 08, 2014.

[2] Li Yongyi, Li Hongjing and Lin Xin, Earthquake relief materials allocation model and method under the condition of material [A].03:2010.

[3] Lin Xin, Study on earthquake relief supplies scheduling and allocation management based on GIS[D], Nanjing University of Technology,2009.

[4] Xu Xiaoyong, Improvement of Matlab language program design of fuzzy clustering analysis algorithm[J]. Journal of Yunnan University of Nationalities (Natrual Science Edition), 196-197+210:03, 2006. 